\title{
Presença de Cryptosporidium spp em crianças com diarréia aguda em uma creche pública de Recife, Estado de Pernambuco
}

\author{
Presence of Cryptosporidium spp in children with acute diarrhea \\ in a public daycare center in Recife, State of Pernambuco
}

\author{
Wheverton Ricardo Correia do Nascimento ${ }^{1}$, Isabella Macário Ferro Cavalcanti ${ }^{1}$, \\ João Inácio Irmão ${ }^{2}$ e Francisca Janaina Soares Rocha ${ }^{2}$
}

\begin{abstract}
RESUMO
0 objetivo do presente estudo foi analisar a frequiência de oocistos de Cryptosporidium spp em amostras fecais de crianças, de 1 a 14 anos, de uma creche pública localizada em uma comunidade carente da cidade do Recife, Pernambuco. A pesquisa foi realizada no período de 28 de junho de 2006 a 3 de abril de 2007, e envolveu 182 crianças. Das amostras analisadas 59 (32,4\%) foram positivas quanto à presença de oocistos de Cryptosporidium spp, e a faixa etária mais acometida foi a de 3 a 5 anos de idade (54,2\%). A alta frequiência de amostras positivas para Cryptosporidium spp obtidas neste estudo comprovam que creches são ambientes propícios a essa ocorrência devido ao contato direto entre criança-criança, crianças e funcionários. A maior via de infecção por Cryptosporidium spp é a transmissão interpessoal, que é bem ilustrada em creches. A imaturidade, deficiências do sistema imune e hábitos higiênicos inadequados são fatores que também contribuem para esse tipo de infecção.
\end{abstract}

Palavras-chaves: Creche. Crianças. Parasita oportunista. Cryptosporidium spp.

\begin{abstract}
The objective of the present study was to analyze the frequency of oocysts of Cryptosporidium spp in fecal samples from children aged one to fourteen years at a public daycare center located in a needy community in the city of Recife, Pernambuco. The investigation was carried out between June 28, 2006, and April 3, 2007, and involved 182 children. Among the samples analyzed, 59 (32.4\%) were positive regarding the presence of oocysts of Cryptosporidium spp, and the age group most affected was between three and five years (54.2\%). The high frequency of samples positive for Cryptosporidium spp obtained in this study confirms that daycare centers are an environment that favors such occurrences, because of the direct contact between children or between children and staff. The most important infection route for Cryptosporidium spp is person-to-person transmission, which is well illustrated in daycare centers. Immaturity, deficiencies of the immune system and inadequate hygiene habits are factors that also contribute towards this type of infection.
\end{abstract}

Key-words: Daycare center. Children. Opportunistic parasite. Cryptosporidium spp.

Indivíduos imunossuprimidos estão sujeitos a uma série de complicações infecciosas causadas por agentes oportunistas, e em decorrência deste fato, poder-se-á observar o aparecimento de algumas doenças denominadas emergentes ${ }^{20}$. Entre os microrganismos emergentes, destacam-se os protozoários entéricos oportunistas, em especial os coccídios (Filo: Apicomplexa) e os microsporídios (Filo: Microsporidia), relatados como responsáveis por inúmeros casos patológicos, apresentando infecções refratárias ou incuráveis, com significativas causas de morte. Os coccídios intestinais de maior importância

1. Acadêmicos do Curso de Biomedicina, Universidade Federal de Pernambuco, Recife, PE. 2. Departamento de Medicina Tropical, Universidade Federal de Pernambuco, Recife, PE.

Endereço para correspondência: Profa. Francisca Janaina Soares Rocha. Deptọ de Medicina Tropical/UFPE. R. Prof. Moraes Correa Rego s/n, Cidade Universitária, 50670-420 Recife, PE.

Tel.: 5581 2126-8525; Fax: 5581 2126-8525

e-mail: janainarocha@ufpe.br

Recebido para publicação em 26/05/2008

Aceito em 20/03/2009 que infectam o trato intestinal humano são: Isospora belli, Cryptosporidium spp, e o mais recentemente descrito, Cyclospora cayetanensi ${ }^{27}$.

o Cryptosporidium spp era considerado um patógeno oportunista que acometia apenas pessoas imunodeprimidas, mas o número de casos em indivíduos imunocompetentes vêm se tornando cada vez mais freqüente. Trata-se, na atualidade, de um patógeno intestinal distribuído em todo o mundo. Esta ocorrência é dependente de fatores que incluem o clima, a idade e outros aspectos demográficos de uma população; em crianças com idade entre 1 a 5 anos com diarréia, o Cryptosporidium spp é frequentemente apontado como o patógeno causador da infecção $0^{28}$.

As doenças ocasionadas por parasitas oportunistas estão intimamente relacionadas a estados imunes deficientes, tais como ocorre nos casos da síndrome da imunodeficiência humana (SIDA), nas doenças linfoproliferativas e em indivíduos transplantados ${ }^{9}$. Porém, as condições sócio-econômicas mais baixas, o estado subnutricional e/ou de desnutrição, o consumo de água não tratada, a falta de saneamento básico, a imaturidade 
do sistema imune e os hábitos higiênicos inadequados são fatores que também podem propiciar os casos de criptosporidiose nos infantes ${ }^{4}$.

As gastroenterites são as principais causas de mortalidade infantil em países desenvolvidos e em desenvolvimento ${ }^{122}$. Vírus, bactérias e parasitas podem ser considerados agentes etiológicos. Com relação aos parasitas, destaca-se o protozoário, Cryptosporidium spp, tanto pelo seu caráter oportunista como pelo fato dos surtos diarréicos, persistentes e de duração variável que ele causa nas crianças. A infecção se dá, geralmente, pela ingestão de água e/ou alimentos contaminados com os oocistos esporulados $^{21242630}$.

As creches, por se tratarem de ambientes fechados, nos quais as crianças ficam a maior parte do dia, passam a ser um fator a mais de exposição às enteroparasitoses. 0 fato de freqüentar creches é mais uma característica de nível sócio-econômico desfavorável. A maior urbanização e a maior participação feminina no mercado de trabalho fizeram com que estes ambientes passassem a ser, depois do ambiente doméstico, o primeiro espaço externo que a criança freqüienta ${ }^{13}$

0 presente trabalho teve como objetivo analisar a freqüência de Cryptosporidium spp em amostras fecais de crianças, de 1 a 14 anos, de uma creche localizada em uma comunidade carente da Cidade do Recife, Pernambuco, além de obter informações sobre as condições de vida dessas crianças e de seus familiares.

\section{MATERIAL E MÉTODOS}

A pesquisa envolveu 182 crianças, com idade entre 1 e 14 anos, de uma creche pública localizada na Várzea, bairro com 64.512 (sessenta e quatro mil, quinhentos e doze) habitantes, na Cidade de Recife, $\mathrm{PE}^{29}$. As amostras foram coletadas no período de 28 de junho de 2006 a 3 de abril de 2007.

0 estudo foi aprovado pelo comitê de ética do Centro de Ciências da Saúde (CCS) da Universidade Federal de Pernambuco (UFPE), CAAE: 0075.0.1.172.000-06, envolvendo somente as crianças, cujos pais e/ou responsáveis leram e assinaram o termo de consentimento livre esclarecido, autorizando a realização do exame coproparasitológico nos seus filhos. Foram distribuídos potes coletores plásticos para a coleta do material, que foram encaminhados ao laboratório pelos funcionários da creche para dar prosseguimento às análises.

As amostras foram analisadas no Laboratório de Parasitologia do Departamento de Medicina Tropical da UFPE. Para cada amostra, foram preparadas duas lâminas. Os ooscistos de Criptosporidium spp foram detectados através da técnica de Ziehl-Neelsen modificada, coloração com fucsina-carbólica (método Kinyoun) ${ }^{12}$, precedida pela técnica de sedimentação espontânea (método de Hoffman, Pons e Janer), utilizado no exame direto das fezes para a pesquisa de cistos de protozoários, bem como ovos e larvas de helmintos ${ }^{1831}$.

No decorrer da pesquisa, foram visitadas 43 casas de famílias que mantêm seus filhos na creche. Com a visita, foram analisados o perfil sócio-econômico e as condições básicas de saneamento ambiental e higiene pessoal dos entrevistados. As informações foram obtidas por meio de questionários padronizados.

De posse dos resultados da pesquisa, houve a entrega dos laudos parasitológicos ao corpo médico da creche, para que fosse providenciado o tratamento das crianças parasitadas.

\section{RESULTADOS}

Das 182 crianças examinadas, $59(32,4 \%)$ foram positivas para Cryptosporidium spp (Figura 1), onde 29 eram meninos e 30 meninas, não demonstrando assim diferença significativa entre os sexos.

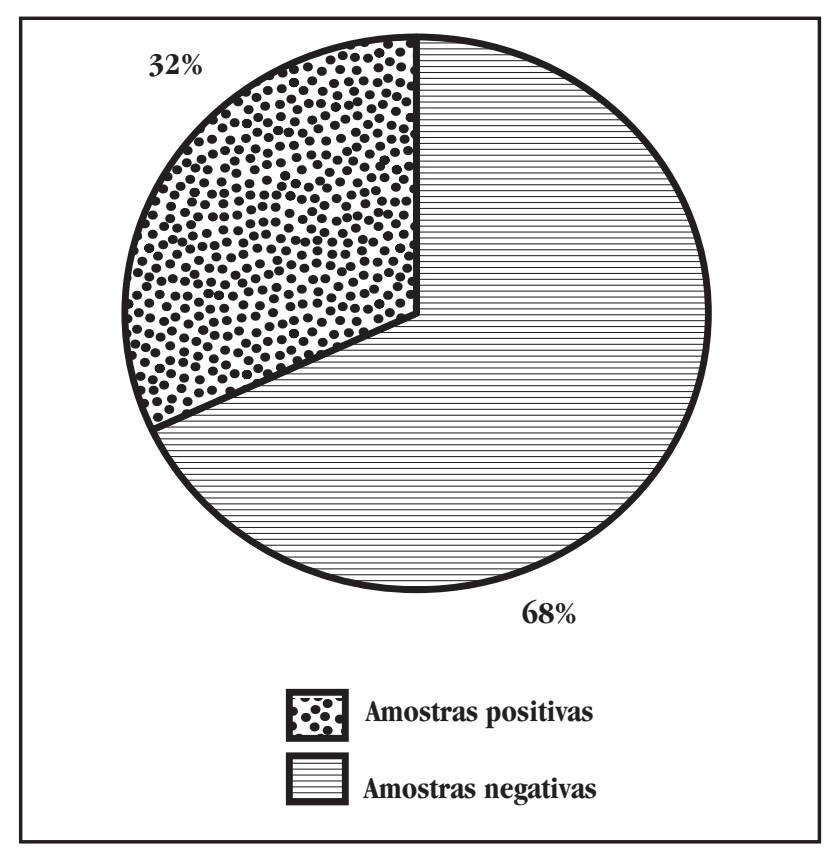

\section{FIGURA 1}

Frequiência das amostras de Cryptosporidium spp em crianças da creche Lar Fabiano de Cristo, coletadas no período de 28 de junho de 2006 a 3 de abril de 2007.

A faixa etária mais acometida foi a de 3 a 5 anos de idade (54,2\%), seguida pela de 0 a 3 anos (23,7\%), 5 a 7 anos (13,6\%), 7 a 9 anos (6,8\%) e 9 a 11 anos (1,7\%). Não foram observados oocistos de Cryptosporidium spp, nas crianças entre 11 e 13 anos de idade (Figura 2).

A infecção por Cryptosporidium spp está freqüentemente associada com episódios diarréicos, persistentes, com duração variável $^{24}$. No presente trabalho, este enteropatógeno foi mais detectado em fezes diarréicas $(84,2 \%)$, tanto líquidas como pastosas em comparação com as fezes consistentes (7,8\%), como mostra a Tabela 1.

A avaliação do questionário aplicado as 43 moradias das crianças em estudo mostrou que apenas $11,6 \%$ dos pais possuíam o ensino médio completo e $27,9 \%$ das famílias tinham renda superior a um salário mínimo. Somente $27,9 \%$ possuíam rede de esgoto, estando as demais vivendo em ambientes com esgoto a céu aberto. Na sua grande maioria (93\%), as casas tinham pragas domésticas, como moscas, ratos e baratas, que poderiam 


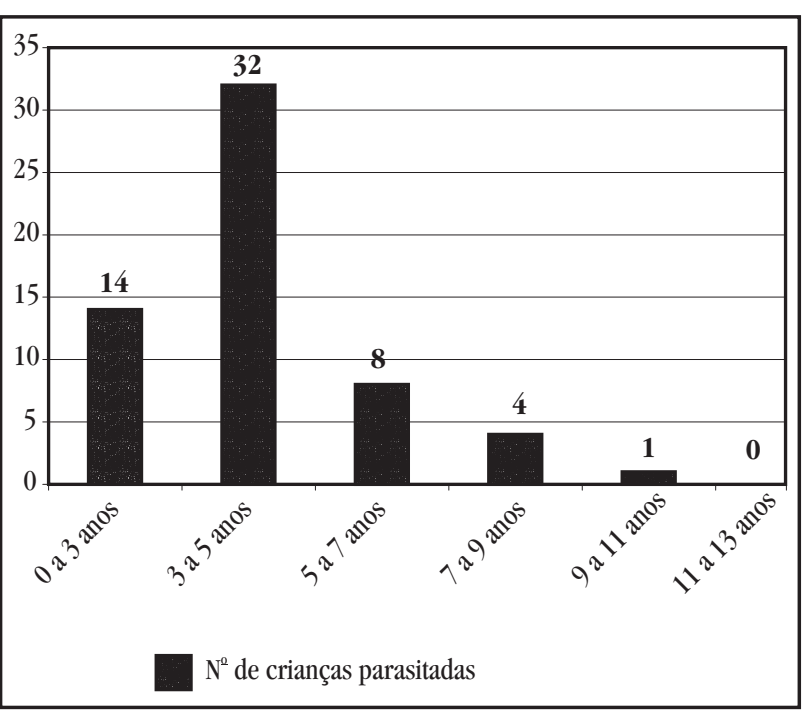

FIGURA 2

Frequiência da detecção de Cryptosporidium spp por faixa etária em crianças da creche Lar Fabiano de Cristo coletadas no período de 28 de junho de 2006 a 03 de abril de 2007 (Cada faixa etária compreende 30 meses - sendo assim de 0 a 3 anos corresponde até a idade de 2 anos e seis meses).

\section{TABELA 1}

Frequiência da detecção de Cryptosporidium spp por consistência das fezes em crianças da creche Lar Fabiano de Cristo coletadas no período de 28 de junho de 2006 a 3 de abril de 2007.

\begin{tabular}{lccccc}
\hline & Amostra & & \multicolumn{2}{c}{ Cryptosporidium spp } \\
\cline { 5 - 6 } Fezes & total $\left(\mathrm{n}^{0}\right)$ & & $\mathrm{n}^{0}$ & $\%$ \\
\hline Líquidas & 59 & & 26 & 44,0 \\
Pastosas & 72 & & 29 & 40,2 \\
Consistentes & 51 & & 4 & 7,8 \\
\hline
\end{tabular}

funcionar como vetores de parasitas. Com relação às crianças, as informações que chamaram a atenção foram: 48,8\% relataram casos de diarréia, 51,6\% já eliminaram vermes nas fezes e 76,7\% brincavam com areia e animais. Quase todas as casas possuíam água encanada e coleta de lixo, ao mesmo tempo em que a maioria informou fazer a correta lavagem de frutas e verduras, assim como lavar as mãos antes das refeições e após usar o banheiro.

\section{DISCUSSÃO}

Um estudo realizado em 1991, mostrou que a prevalência da criptosporidiose em pacientes portadores da SIDA, em países desenvolvidos, é em torno de 10 a 20\%. Já em análises realizadas em amostras de fezes de pacientes com gastroenterites, a prevalência foi de 1 a 4\% na Europa e América do Norte e de 3 a 20\% na África, Ásia, Austrália, América Central e do Sul7 .

Estudos realizados em diferentes estados no Brasil têm demonstrado uma prevalência de Cryptosporidium spp em crianças (0 a 12 anos de idade) de 4,2\%, 3,3\% e 1,8\% em Uberlândia (MG) ${ }^{11}$, Rio de Janeiro (RJ) ${ }^{30}$ e Ribeirão Preto $(\mathrm{SP})^{22}$, respectivamente.

0 registro da soroprevalência é geralmente alto nos portadores assintomáticos, por volta de 25 a $35 \%$ em países desenvolvidos e de até $95 \%$ naAmérica latina ${ }^{3}$, inclusive emidosos ${ }^{14} 32$, em trabalhadores rurais $^{17}$ e em creches ${ }^{14}$. 0 número de portadores assintomáticos é determinado através de estudos coproparasitológicos, e são geralmente muito baixos (<1\%) em países desenvolvidos 7 . Embora tenha exibido altos índices em creches ${ }^{6} 1015$.

Os dados obtidos, através do presente estudo, mostraram que das 182 amostras analisadas, 32\% foram positivas para Cryptosporidium spp, uma frequiência consideravelmente alta em relação àquelas observadas em outros estudos realizados no Brasil ${ }^{112230}$.

Sugere-se, então, que a maior vulnerabilidade das crianças à criptosporidiose possa ocorrer devido a alguns fatores, como: permanência em tempo integral ou semi-integral na creche, ter menos de 5 anos (Figura 2), pouca capacidade de deslocamento e maior contato pessoa-pessoa ${ }^{19}$, o que facilitaria a manutenção do ciclo.

Ainda que não tenha sido possível estabelecer o período de duração do episódio diarréico no presente estudo, é coerente supor que, nesta fase haja intensa eliminação de oocistos nas fezes ${ }^{30}$, fato este comprovado pela baixa freqüência de Cryptosporidium spp detectados nas amostras de fezes do tipo consistente (Tabela 1).

o quadro diarréico observado em $84 \%$ das crianças pode também ter sido ocasionado por outros parasitas intestinais, como: Giardia lamblia e/ou Entamoeba histolytica/dispar, associados ou não ao Criptosporidium spp. Por exemplo, das amostras diarréicas, 43\% foram positivas para Giardia lamblia ${ }^{23}$.

Em indivíduos imunocompetentes, a criptosporidiose é limitada por uma resposta imune claramente direcionada contra 0 parasita. Nas infecções em pacientes com resposta imune celular deficitária, por exemplo, nos casos de falhas congênitas, SIDA ou uso de quimioterapia, ou ainda na resposta imune humoral defeituosa, como na hipergamaglobulinemia congênita, a infecção é mais intensa e persistente. Alguns estudos em animais sugerem ainda que a resposta imune possua a capacidade de impedir a ocorrência de re-infecções ${ }^{32}$.

Indivíduos com a imunidade adequada, a este coccídio, são apontados como portadores assintomáticos e disseminadores em países com altas prevalências. Além disso, um estudo realizado com voluntários revelou que a exposição ao mesmo parasita, após um ano de infecção, produziu um quadro significativamente mais leve; a ocorrência da diarréia foi similar em ambas as exposições, mas a gravidade da doença foi menor nos voluntários re-infectados, revalidando assim a teoria da imunoproteção contra re-infecções ${ }^{26}$.

A alta frequiência de amostras positivas para Cryptosporidium $s p$ comprovam que creches são ambientes propícios a essa ocorrência devido ao contato entre crianças, crianças e funcionários, que são muitas vezes mal orientados quanto às medidas de higiene e manipulação de alimentos. A maior via de infecção por Cryptosporidium spp é a transmissão interpessoal, que é bem ilustrada em creches $^{258}$, embora os oocistos sejam frequentemente encontrados em água potável ${ }^{16}$. A imaturidade e deficiências do sistema imune (possivelmente ocasionados por subnutrição) e hábitos higiênicos inadequados são fatores que também podem propiciar infecções deste gênero ${ }^{15}{ }^{32}$, bem evidenciados nos dados obtidos pelos questionários aplicados. 


\section{AGRADECIMENTOS}

Agradecemos à Creche Lar Fabiano de Cristo por disponibilizarem as crianças para a coleta do material e ao laboratório de Parasitologia do Departamento de Medicina Tropical da Universidade Federal de Pernambuco, pelo auxílio na realização das análises.

\section{REFERÊNCIAS}

1. Alverca VO, Gomes TS, Silva MLR, Domingues ALS, Santos NOS. Incidência de gastrenterite infantil de etiologia viral em Juiz de Fora, Minas Gerais, no período de janeiro a dezembro de 1998. Jornal Brasileiro de Patologia 4:219-227, 2000 .

2. Casemore DP. Epidemiological aspects of human cryptosporidiosis. Epidemiology and Infection 104:1-28, 1990

3. Casemore DP, Wright SE, Coop RL. Cryptosporidiosis-human and animal epidemiology. In: Fayer R (ed) Cryptosporidium and cryptosporidiosis. CRC Press Boca Raton, FL, p.65-92, 1997.

4. Castro AZ, Viana JDC, Penedo AA, Donatele DM. Levantamento das parasitoses intestinais em escolares da rede pública na cidade de Cachoeiro do ItapemirimES. Newslab 64:140-144, 2004

5. Cordell RL, Addiss DG. Cryptosporidiosis in child care settings: a review of the literature and recommendations for prevention and control. Pediatric Infectious Disease Journal 13:310-317, 1994.

6. Crawford FG, Vermund SH. Human cryptosporidiosis. Critical Reviews in Microbiology 16:113-159, 1988

7. Current WL, Garcia LS. Cryptosporidiosis. Clinical Microbiology Reviews 4:325-258, 1991

8. Fayer R, Ungar BLP. Cryptosporidium spp and cryptosporidiosis. Microbiological Reviews 50:458-483, 1986

9. Ferreira MS. Infections by Protozoa in Immunocompromised Hosts. Memórias do Instituto Oswaldo Cruz 95 (supl I):159-162, 2000

10. Garcia-Rodriguez JÁ, Martin-Sánchez AM, Blasco CA, Cedeño-Montaño J, Pedro HMI. The incidence of cryptosporidiosis in children: a one year prospective survey in a general hospital in Spain. European Journal of Epidemiology 5:70-73, 1989 .

11. Gennari-Cardoso ML, Costa-Cruz JM, Castro E, Lima LMFS, Prudente DV. Cryptosporidium sp in children suffering from acute diarrhea at Uberlândia city, State of Minas Gerais, Brazil. Memórias do Instituto Oswaldo Cruz 91:551-554, 1996

12. González-Ruiz A, Bendall R. Size matters: the use of the ocular micrometer in diagnostic parasitology. Parasitology Today 11:83-85, 1995.

13. Gurgel RQ, Cardoso GS, Silva AM, Santos LN, Oliveira RCV. Creche: ambiente expositor ou protetor nas infestações por parasitas intestinais em Aracajú, SE. Revista da Sociedade Brasileira de Medicina Tropical 38:267-269, 2005.

14. Kuhls TL, Mosier DA, Crawford DL, Griffis J. Seroprevalence of cryptosporidial antibodies during infancy, childhood, and adolescence. Clinical Infectious Diseases 18:731-735, 1994.
15. Lacroix C, Berthier M, Agius G, Bonneau D, Pallu B, Jacquemin JL. Cryptosporidium oocysts in immunocompetent children: epidemiological investigations in the day care centres of Poitiers, France. European Journal of Epidemiology 3:381-385, 1987.

16. LeChevallier MW, Norton WD, Lee RG. Occurrence of Giardia and Cryptosporidium spp in surface water supplies. Applied and Environmental Microbiology 56:2610-2616, 1991

17. Lengerich EJ, Addiss DG, Marx JJ, Ungar BL, Juranek DD. Increased exposure to cryptosporidia among dairy farmers in Wisconsin. Journal of Infectious Diseases $167: 1252-1255,1993$

18. Lutz A. O Schistosomum mansoni e a schistosomatose, segundo observações feitas no Brazil. Memórias do Instituto Oswaldo Cruz 11:124-155, 1949.

19. Macedo LMC, Costa MCE, Almeida LM. Parasitismo por Ascaris lumbricoides em crianças menores de dois anos: estudo populacional em comunidade do Estado do Rio de Janeiro. Caderno de Saúde Pública 15:173-178, 1999.

20. Manzi RS, García-Zapata MTA. Diagnóstico laboratorial dos protozoários entéricos oportunistas em Goiânia, GO. Revista da Sociedade Brasileira de Medicina Tropical 33:597-598, 2000.

21. Marshall MM, Naumovitz D, Ortega Y, Sterling CH. Waterborne protozoan pathogens. Clinical Microbiology Reviews 10:67-85, 1997.

22. Medeiros MIC, Neme SN, Silva P, Capuano DM, Errera MC, Fernandes AS Valle GR, Avila FA. Etiology of acute diarrhea among children in Ribeirão Preto, Brazil. Revista do Instituto de Medicina Tropical de São Paulo 43:21-24, 2001.

23. Nascimento WRC, Cavalcanti IMF, Medeiros CSQ, Alencar AA, Brandão JOC, Braz SCM Gomes RAF, Silva CES, Irmão JI, Rocha FJS. Parasitoses intestinais: prevalência de protozoários em uma creche comunitária da região metropolitana do Recife-PE, 2007. In: Resumos do XX Congresso Brasileiro de Parasitologia, Recife p. 21, 2007.

24. Newman RD, Sears CL, Moore SR, Nataro JP, Wuhib T, Agnew DA Guerrant RL, Lima AAM. Longitudinal study of Cryptosporidium infection in children in northeastern Brazil. The Journal of Infectious Diseases 180:167-175, 1999.

25. Nichols GL. Food-borne protozoa. British Medical Bulletin 56: 209-235, 2000.

26. Okhuysen PC, Chappell CL, Sterling CR, Jakubowski W, DuPont HL. Susceptibility and serological response of healthy adults to reinfection with Cryptosporidium parvum. Infection and Immunity 66:441-443, 1998.

27. Ortega YR, Sterling CR, Gilman RH, Cama VA, Diaz F. Cyclospora species - a new protozoan pathogen of humans. New England Journal of Medicine 328:13081312, 1993.

28. Palmer SR. Epidemiological methods in the investigation of food poisoning outbreaks. Letters in Applied Microbiology 11:109-115, 1990.

29. Pernambuco de A/Z. Bairros do Recife-Varzea. 22 out. 2005. Disponível em $<$ http://www.pe-az.com.br/bairros_recife/bairros_varzea.htm> Acessado em: 22 de fevereiro, 2008

30. Silva S, Silva SP, Gouveia YS, Silva NO, Melo MERM, Moura H, Neves RH, Bello AR, Machado-Silva, JR. Ocorrência de Cryptosporidium sp em amostras fecais de crianças, menores de 10 anos de idade, com indicação clínica de Rotavírus Revista da Sociedade Brasileira de Medicina Tropical 36:421-423, 2003.

31. Vallada EP. Manual de Exame de Fezes. Editora Atheneu, São Paulo, p.119-120, 1997.

32. Zu SX, Fang GD, Fayer R, Guerrant RL. Cryptosporidiosis-pathogenesis and immunology. Parasitology Today 8:24-27, 1992 